\section{PLANO DIRETOR \\ PARA O DESENVOLVIMENTO DA \\ EPIDEMIOLOGIA NO BRASIL \\ 2000-2004}

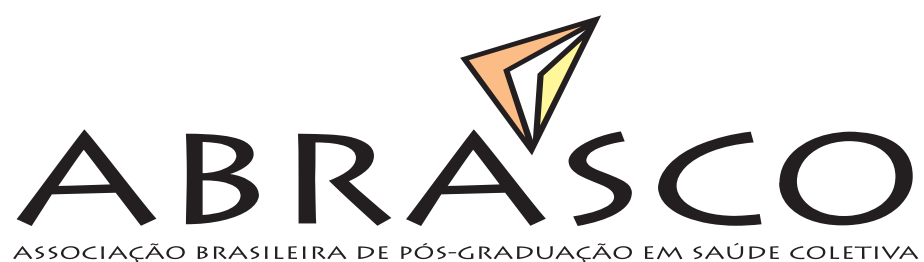

\section{Comissão de Epidemiologia}

\author{
Rio de Janeiro, 2000
}

Diretoria da Abrasco 2000-2003

José Carvalho de Noronha - UERJ - Presidente Francisco Eduardo Campos - UFMG - Vice Presidente Jairnilson Paim - UFBA - Vice Presidente Márcia Furquim de Almeida - USP - Vice Presidente Paulo Mangeon Elias - USP - Vice Presidente Paulo Marchiori Buss - FIOCRUZ - Vice Presidente

\section{Secretaria Executiva}

Péricles Silveira da Costa - Secretário Executivo Álvaro Hideyoshi Matida - Secretário Executivo

Conselho 2000 - 2003

Depto. de Medicina Social / FMC

Santa Casa de São Paulo (Regina Maria Marsiglia)

Escola de Saúde Pública / SES-RS

(Ricardo Burg Ceccin)

Núcleo de Estudos de Saúde Coletiva CCS / UFRJ (Roberto A.Medronho)

Núcleo de Estudos de Saúde Coletiva PE / DSC / CpqAM / FIOCRUZ (Lia A. Giraldo)

Núcleo de Estudos de Saúde Pública CEAM / UNB (Ana Maria Costa)

\section{Comissão de Epidemiologia} Coordenadora:

Maria do Carmo Leal - ENSP/FIOCRUZ Componentes:

Chester L. Galvão César - FSP/USP

Gulnar de Azevedo e Silva Mendonça - IMS/UERJ

José da Rocha Carvalheiro - CIP/SES/SP José Eluf Neto - DMP/FM/USP

Lígia Kerr Pontes - DSC/FM/UFCE

Luiz Augusto Facchini - DMC/UFPel

Maria da Glória Teixeira - ISC/UFBA

Maria Fernanda Lima e Costa - DMPS/FM/UFMG

RELATORES

\section{DOS GRUPOS DE TRABALHO}

Grupo de Ensino

Marilisa Berti de Azevedo Barros - Unicamp Luiz Roberto Oliveira - Unesp Diana Maul de Carvalho - UFRJ

\section{Grupo de Pesquisa} Lígia Regina Kerr Pontes - UFCE Michael Reicheinhein - IMS/UERJ

César Victora - UFPel Ricardo Arraes Ximenes - UFPE 21.041-210 - Manguinhos - Rio de Janeiro - RJ Tel.: (0XX21) 598-2527

Tel./Fax: (0XX21) 560-8699 e 560-8403

E-mail: abrasco@ensp.fiocruz.br Home Page: www.abrasco.org.br

\section{Apoio}

Centro Nacional de Epidemiologia/Fundação Nacional de Saúde/Ministério da Saúde CENEPI/FUNASA/MS

Revisão

Ana Tereza de Andrade
Grupo de Políticas, Programas e Serviços de Saúde Zulmira Maria de Araújo Hartz - FIOCRUZ Henrique Leonardo Guerra - CPqRR/MG Marcos Drumond - Proaim/SP

\section{Coordenação Editorial} Gulnar de Azevedo e Silva Mendonça - IMS/UERJ Maria da Glória Teixeira - ISC/UFBA Álvaro Hideyoshi Matida - Abrasco 


\section{Sumário}

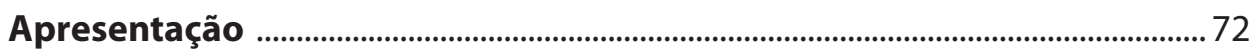

O Ensino da Epidemiologia ........................................................................................

Nível da Pós-graduação - stricto sensu ............................................................. 73

Nível da especialização/capacitação e residência -

pós-graduação lato sensu ...................................................................................

Nível da Graduação ..................................................................................................

A Pesquisa em Epidemiologia …………………………………………………....

Política de Desenvolvimento Científico e Tecnológico

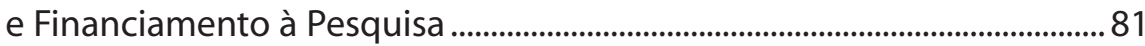

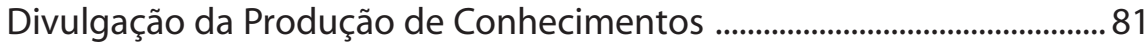

Questões Gerais .......................................................................................................... 82

\section{A Epidemiologia nas Políticas, Programas e Serviços}

de Saúde ....................................................................................................................... 82

Sistemas de Informação de Interesse Epidemiológico .................................... 86

Desenvolvimento e Utilização de Metodologias para

Análise das Situações e das Intervenções em Saúde ....................................8 87

Práticas Epidemiológicas nos Programas e Serviços ......................................8 88

Políticas de Recursos Humanos ............................................................... 88

Vigilância em Saúde .................................................................................... 88

Avaliação em Saúde ................................................................................... 89

Inserção nas Políticas Intra e Intersetoriais .......................................................8 89

\section{Anexos}

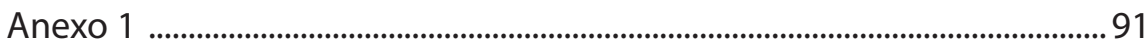

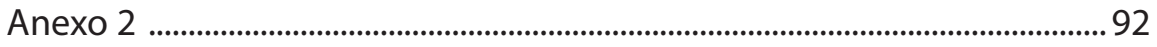




\section{Apresentação}

Este plano diretor vem dar seqüência ao processo iniciado pelos dois planos anteriores para o desenvolvimento da Epidemiologia no Brasil. Sua elaboração se deu em algumas etapas que tiveram como marco o Seminário realizado em Brasília, de 02 a 04 de agosto de 2000, organizado pela Comissão de Epidemiologia da Abrasco e promovido pelo Cenepi/Ministério da Saúde (Anexo 1). Neste seminário participaram 59 epidemiologistas representativos dos programas de ensino e pesquisa do país e dos serviços de saúde das esferas municipal, estadual e federal (Anexo 2).

Os participantes do seminário receberam inicialmente o II Plano Diretor e o documento elaborado por epidemiologistas que participaram da definição dos planos anteriores e que teve como objetivo tecer comentários sobre a aplicação do mesmo no período a que foi proposto.

Durante o seminário, os participantes, após apresentação dos temas estratégicos definidos a partir de inserções de atuações específicas (ensino, pesquisa e serviços), foram divididos em grupos de trabalho. Esses grupos avaliaram e elaboraram propostas que foram levadas para um relatório, propiciando a versão preliminar do plano, avaliada pela Comissão de Epidemiologia e por todos os participantes do Seminário Nacional. Assim, foi possível ser concluída a versão final do plano.

A continuidade deste trabalho, iniciado em 1990 por iniciativa da Comissão de Epidemiologia da Abrasco, vem estimulando o desenvolvimento nacional da epidemiologia a partir da soma de esforços na organização de estratégias de criação e implementação de ações de intervenção coordenadas. O registro sistemático dessas ações demonstra a consolidação e expansão da epidemiologia, tanto na área acadêmica quanto nos serviços de saúde. Embora ainda permaneçam algumas lacunas e pontos de maior fragilidade, a comparação entre os planos permite constatar que os ganhos alcançados foram muitos.

Esperamos, assim, que as sugestões e propostas aqui contidas possam embasar e estimular o trabalho dos epidemiologistas de forma agregadora nos diversos campos de atuação dentro da perspectiva de fortalecimento da saúde pública e da garantia de compromisso com melhores condições de vida para a população brasileira.

Comissão de Epidemiologia da Abrasco 


\section{O Ensino da Epidemiologia}

Para discutirmos o ensino e o Plano Diretor da Epidemiologia em sua totalidade, torna-se necessário considerar o contexto mais amplo das políticas de saúde e de educação do país, no qual as questões aqui levantadas se inserem.

No âmbito da saúde, devemos assumir o compromisso com a construção do SUS, servindo de eixo norteador do Plano Diretor de Epidemiologia, especialmente no que diz respeito aos processos de municipalização e reorganização dos serviços. Afinal, a complexidade das situações de saúde no país, na maioria dos exemplos causadas pelas medidas macroeconômicas de ajustes, vem sendo agravada pela extrema iniqüidade social, gerando demandas e desafios à organização dos serviços e à formação de profissionais.

No processo de reorganização dos serviços, observam-se várias mudanças na legislação, programas e normas (NOBs) relativas à saúde. Esse fato tem provocado uma intensa reordenação das ações de estados e municípios e diferenciadas demandas ao ensino da Saúde Pública e da Epidemiologia, haja vista a proposta do Programa de Saúde da Família que, propiciando mudanças na reorganização dos serviços, tem gerado novas necessidades de formação, envolvendo progressivamente um conjunto cada vez maior de instituições formadoras.

As políticas de recursos humanos apresentam lacunas no campo das profissões que geram obstáculos às propostas de incremento na área de formação dos recursos humanos. Entre elas, destacam-se: a ausência de definições de carreira, os baixos níveis salariais e o reduzido número de oportunidades efetivas de formação e aprimoramento profissional - questões que, seguramente, fazem parte desse contexto.

No âmbito da educação, também devem-se considerar alguns cenários adversos, no que diz respeito às diretrizes e financiamento do ensino de pós-graduação lato sensu e stricto sensu (capacitação e doutorado/mestrado), tais como: a nova
$\mathrm{LDB}$, as normas de reconhecimento das universidades, a política de financiamento da Capes, que sinaliza reforço ao doutorado, desincentivo dos programas de mestrado e proposta de mestrado profissionalizante.

Em relação ao ensino da Epidemiologia em todos os níveis de formação, reafirmase a desejável indissociabilidade do ensino, pesquisa e práticas de serviços. De outro lado, é possível identificar avanços recentes da Epidemiologia, especialmente quanto à análise de situações de saúde, de séries temporais e espaciais e desenvolvimento de metodologias de avaliação apontando para a incorporação de novos conteúdos na reformulação do ensino.

As inovações tecnológicas, não somente na área da informática e da informação, como dos processos pedagógicos (PBL etc.) e novas modalidades de ensino, como cursos seqüenciais, ensino à distância e semipresenciais, também compõem o contexto de nossos problemas e propostas.

Por último, é preciso considerar que todo processo de ensino deve apontar para cenários futuros da atuação profissional, além de responder às exigências do presente.

Tendo em vista a diversidade da natureza dos aspectos que envolvem o ensino da epidemiologia e suas diferentes formas de inserção, foram levantados alguns aspectos, sendo identificados problemas nos três níveis do ensino da epidemiologia. Em seguida, apresentamos as proposições de caminhos para solucioná- los.

\section{Nível da pós-graduação - Stricto sensu}

O ensino de pós-graduação stricto sensu é o mais bem diagnosticado e acompanhado pela área. $\mathrm{O}$ amplo processo de avaliação da PG em Saúde Coletiva, desenvolvido nos anos 1995 e 1996, gerou significativa consolidação de dados e possibilitou importante aprofundamento no entendimento das características do ensino e produção científica da área. Reunião específica sobre "Ensino e pesquisa de Epidemiologia nos cursos de pós-graduação estrito senso", realizada em 
1996, atendendo à proposta do $2^{\circ}$ Plano Diretor, apontou as questões mais prementes.

O Comitê de Avaliação da Área de Saúde Coletiva da Capes, de 1996/97¹ , reconhece o crescimento quantitativo e qualitativo da área. Entre os aspectos levantados no relatório, verificaram-se: maior proporção de docentes com doutoramento; número de orientandos por orientador, por vezes excessivo; aumento do volume de produção científica, mas ainda insuficiente publicação em revistas Qualis A e B; concentração da produção em alguns docentes; insuficiente produção e publicação dos alunos; delimitação e consolidação de linhas temáticas ainda insuficientes; redução do tempo médio de defesa de teses, mas ainda excessivamente prolongado; grande heterogeneidade do dinamismo dos cursos na atualização científica de docentes e na renovação dos quadros; aumento das atividades de intercâmbio entre instituições nacionais e internacionais, mas em níveis ainda insuficientes; poucas iniciativas de monitoramento da qualidade dos cursos; necessidade de registrar como indicadores a publicação das teses defendidas e o número de bolsas-sanduíche efetivadas.

Verificou-se, também, aprimoramento no processo de avaliação da Capes. Procedimentos e critérios têm sido discutidos em reuniões do representante da Área de Saúde Coletiva da Capes com os coordenadores dos programas de Saúde Coletiva, em processo não usual em outras áreas científicas. Considera-se a necessidade de avançar nesse processo participativo e crítico.

\section{Problemas}

- Insuficiente produção científica do corpo docente e discente; demora na defesa da tese; insuficiência de financiamento dos cursos, poucos recursos para bolsas de estudo e para realização de pesquisas; insuficiente atualização do corpo docente; pouco intercâmbio de docentes e alunos entre os cursos e de mecanismos de avaliação das disciplinas e dificuldades especiais dos cursos emergentes.

- Problemas quanto à relevância e ao peso dos conteúdos de epidemiologia, nos cursos de Saúde Coletiva, e conteúdo geral de Saúde Coletiva, nos cursos ou áreas de Concentração de Epidemiologia, e ao conteúdo mínimo de Bioestatística, em curso de PG de Epidemiologia.

- Dificuldades dos programas em responder às necessidades de formação dos profissionais vinculados aos serviços. Destacam-se a duração muitas vezes excessiva; o impedimento de recebimento de bolsas pelo profissional, em decorrência do vínculo empregatício; a falta de apoio da instituição de origem, que às vezes não aproveita o próprio resultado do estudo.

- Insuficiente discussão pelo conjunto da comunidade científica da área de epidemiologia quanto à proposta do mestrado profissionalizante. Essa modalidade de mestrado, que poderá responder de forma mais adequada às demandas de formação para os serviços de saúde, necessita de discussão mais aprofundada, de divulgação de propostas e de avaliação de experiências.

- Tempo reduzido de formação; inexistência de financiamento pela Capes e a conseqüente necessidade de fontes alternativas não regulares; a falta de clareza na diferenciação em relação à especialização e quanto ao efetivo reconhecimento de equivalência com o mestrado acadêmico.

- Necessidade de reavaliação do tipo de produto final (monografia, dissertação, artigo científico, produto tecnológico) desejável nas diferentes modalidades de formação pós-graduada.

1 A avaliação periódica a que os programas de PG são submetidos, sob responsabilidade do Comitê de Avaliação da Área da Saúde Coletiva da Capes, resulta em relatórios que sintetizam os aspectos de progresso e de insuficiências. 
Proposições

- Realização de fóruns promovidos pela Abrasco para discussão dos problemas e aprimoramento do sistema de pósgraduação em saúde coletiva.

- Realização, no decorrer do próximo qüinqüênio, de um novo, abrangente e completo diagnóstico da pós-graduação em saúde coletiva no país.

- Divulgação e discussão do mestrado profissionalizante em fóruns promovidos pela Comissão de Epidemiologia da Abrasco, além da oficina do próximo congresso e ampliada além do âmbito dos coordenadores dos cursos de pósgraduação.

- Discussão desse ponto na pauta de fóruns que serão programados para discutir a pós-graduação.

\section{Nível da especialização/capacitação e} residência-pós-graduação lato sensu

Durante os anos de 1992-1995, ocorreu um acúmulo significativo de experiências nas modalidades de ensino de capacitação / especialização no interior da programação de capacitação em epidemiologia do Cenepi/MS. Instituições de todas as regiões brasileiras, contando com apoio para ampliação de infra-estrutura, ofereceram vários cursos com durações, conteúdos e estratégias de ensino diferenciados.

O processo de avaliação do programa permitiu identificar as principais distorções e apontar caminhos para redirecionar e aprimorar as estratégias de capacitação. Mais recentemente, multiplicaram-se programas de capacitação em DST/AIDS, patrocinados por convênios das universidades com o Ministério da Saúde, envolvendo colaboração importante da disciplina de epidemiologia. Dados da Unidade de Treinamento da Coordenação Nacional de DST/AIDS registram a realização, em 1996, de 61 cursos de treinamento e capacitação em Epidemiologia e Vigilância Epidemiológica, com 957 vagas, oferecidos por 9 instituições; de 37 com 718 vagas, em 1997; e de 20 cursos com 539 vagas, em 1998.
A atual proposta de criação, pelo Cenepi-MS, de uma Rede de Formação para o Sistema Nacional de Vigilância em Saúde, deverá imprimir um novo e significativo avanço no desenvolvimento da disciplina no país, com inovações no processo formativo e impactos na pesquisa e práticas epidemiológicas.

Com esse acúmulo significativo de experiências, avançou-se na compreensão da necessidade de maior participação dos serviços de saúde (fonte principal da clientela) junto às instituições formadoras, sobretudo no que diz respeito à definição de prioridade de cursos, de seleção de conteúdos e estratégias de ensino. Também está amplamente reconhecido o papel central da seleção da clientela para o sucesso dos cursos, merecendo-se destacar os seguintes aspectos: tipo de inserção, perfil, potencial e oportunidade de aplicação efetiva das práticas e conceitos desenvolvidos nos programas de capacitação.

Quanto à formação em programas de residência, detecta-se que continua a crise apontada nos planos anteriores, persistindo, em geral, pouca demanda para as vagas disponíveis. No entanto, a questão da Residência se reatualiza com os incentivos do governo aos programas de Saúde da Família e à demanda por formações específicas. Considera-se que os programas de residência médica exclusiva e os de residência ampliada para outros profissionais de saúde merecem análises diferenciadas pelo contexto atual e especificidade de problemas.

\section{Problemas}

- Falta de avaliações consolidadas das experiências de capacitação/especialização desenvolvidas no país: modalidades utilizadas; conteúdos predominantes; perfil de docentes; formas de seleção de clientela; situação de egressos; existência de mecanismos de educação continuada e das experiências de troca de informações.

- Embora se identifique na conjuntura atual dos serviços de saúde a necessidade 
de formação de recursos humanos, observam-se problemas de absorção desses recursos formados pela falta de definição de carreiras, baixos salários etc..

- Problemas quanto às diferentes formas de capacitação do pessoal dos serviços, realizadas muitas vezes pelo próprio serviço, sendo observada, na maioria dos casos, a necessidade de revisar a adequação de formação de 'multiplicadores'.

- Reconhecimento de novas necessidades para a formação de profissionais em Saúde Coletiva/Epidemiologia, o que implicará agregar, além dos conhecimentos específicos e de consistente visão crítica, a necessidade de desenvolvimento de outras habilidades, como gestão, captação de recursos para projetos e pesquisas etc..

- Insuficiente discussão e aprofundamento sobre as questões relativas à Residência na Área de Saúde Coletiva e necessidade de reavaliação das propostas, tendo em vista a crise que vem afetando os programas de residência em Medicina Preventiva, em Medicina Geral e Comunitária e os feitos e desdobramentos, para essas modalidades, da criação das Residências em Saúde da Família.

\section{Proposições}

- Organização do site para divulgação dos cursos em oferta no país.

- Grupo de trabalho que consolide as características das várias experiências e desenvolva processos de avaliação, definindo metodologias, em especial, instrumentos e indicadores de impacto dos cursos nos serviços de saúde.

- Ação política da Abrasco em relação às questões de carreira e mercado de trabalho dos profissionais do SUS.

- Diagnóstico da possível clientela para todas as modalidades de formação (treinamento, atualização, especialização etc.).

- Revisão dos critérios de necessidades de formação.
- Realização de grupo ou oficina de trabalho para analisar experiências em curso e reavaliar critérios e procedimentos para a formação do profissional que estará habilitado para desenvolver treinamento de técnicos.

- Incluir essa questão nos fóruns correspondentes.

- Realização, pela Abrasco, de um seminário específico sobre a residência na área.

- Estudo avaliando a tendência do número de serviços credenciados, de vagas, da demanda de candidatos, das diretrizes, objetivos e conteúdos teórico e práticos das residências em atividade, da contextualização dos programas frente às mudanças no mercado de trabalho e às necessidades de recursos humanos para a adequada implantação do SUS.

\section{Nível da graduação}

Desde a Primeira Reunião Nacional sobre Ensino e Pesquisa em Epidemiologia, realizada em 1984, o ensino de epidemiologia não tem sido objeto de uma discussão mais detalhada e atualizada de seus conteúdos, tendo em vista o processo de reformas em curso, seja no campo da saúde ou da educação. Contudo, deve-se ressaltar que, nos últimos anos, o interesse pela área nas escolas médicas tem sido crescente. Tal interesse se deve, em grande medida, à extensiva divulgação nessas escolas de temas que se relacionam com a epidemiologia clínica, a medicina com base em evidência e estudos de metanálise.

Além de relevante desenvolvimento técnico-científico da Epidemiologia, alguns temas específicos vêm despertando interesse, não somente nos órgãos de ensino médico, mas na população, na medida em que são amplamente difundidos pelos veículos de comunicação existentes, entre os quais se destacam as questões relativas aos fatores de risco por doenças crônicas. $\mathrm{O}$ atual perfil sanitário do nosso país é marcado por uma forte presença de antigas e novas endemias e a ocorrência de epidemias, apontando para a relevância do conheci- 
mento epidemiológico na determinação de cada um desses quadros.

Na área da informação, a utilização do EPIInfo tem possibilitado a dinamização de grandes bancos de dados de base nacional, seja aprimorando a coleta e uso pelos serviços na instância municipal, proporcionando maior acesso e uso de análises, seja facilitando o ensino da epidemiologia e da estatística, ampliando o acesso à informação e comunicação pela Internet. Por último, as novas tecnologias de ensino e as reformas curriculares em andamento, também colocam novas possibilidades e desafios ao ensino da epidemiologia nos programas de graduação.

\section{Problemas}

- Necessidade de aprofundar a discussão sobre algumas questões centrais do ensino de epidemiologia na graduação: temas privilegiados no conteúdo básico; ênfase em enfoque clínico ou saúde coletiva; ênfase no conteúdo formativo ou instrumental; relação com outras disciplinas da área de saúde coletiva e com disciplinas clínicas; atividades práticas e exercícios; práticas epidemiológicas; uso de novas tecnologias de ensino e de novos enfoques pedagógicos - mudanças que vêm sendo introduzidas.

- Necessidade de aprimoramento e divulgação de material de ensino.

- Necessidade de desenvolvimento de novas tecnologias e práticas pedagógicas.

- Estabelecimento de incorporação do ensino de epidemiologia e bioestatística nos níveis de graduação e pós-graduação.

\section{Proposições}

- Realização de seminário sobre o ensino da epidemiologia da graduação.

- Realização de estudo ou oficina de trabalho para diagnóstico adequado da situação atual e dos problemas do ensino na graduação.
- Produção de livros, de coletânea de exercícios, ampliação do uso da informática (listas de discussão, monitoria virtual).

- Oportunidade de cursos para atualização e capacitação dos docentes com novos métodos e técnicas.

- Mecanismos de divulgação, troca de experiências e de intercâmbio: lista de discussão, homepages etc.

- Discussão do tema nos fóruns que sejam desenvolvidos para discussão de graduação e pós-graduação.

\section{A Pesquisa em Epidemiologia}

O desenvolvimento da pesquisa epidemiológica no Brasil mostra uma vitalidade que pode ser expressa de diferentes maneiras. Uma delas é o crescente número de novos cursos de pós-graduação credenciados e em funcionamento na última década, inclusive fora da região Sudeste, tradicionalmente detentora da maior parcela de cursos de pós-graduação.

Outra maneira pela qual se pode observar o crescimento da área é o aumento do número de grupos de pesquisadores em atuação, passível de ser captado por meio do Diretório de Grupos de Pesquisa do Brasil (DGPB), do CNPq. Entre 1995 e 2000, os grupos de pesquisa em saúde coletiva aumentaram em $115 \%$, enquanto o número total de grupos cresceu $60 \%$ (DGPB, 2000, dados preliminares). Hoje, a Saúde Coletiva é a $8^{\mathrm{a}}$ área do conhecimento quanto ao número de grupos de pesquisa. Observou-se, também, o estabelecimento e diversificação de linhas de pesquisa em diferentes regiões, evidenciando um processo de descentralização da pesquisa epidemiológica no país.

Um outro modo de se avaliar os novos rumos da epidemiologia é verificando o importante aumento do número de publicações científicas, resultantes de pesquisas na área de saúde. Estudo realizado na América Latina ${ }^{2}$ mostrou que Argentina, Brasil, Chile, Cuba, México e Venezuela são res-

2 Pellegrini Filho A, Goldbaum M, Silvi J.Production of scientific articles on health in six Latin American countries, 1973-1992. Rev Panam Salud Publica 1997; 2(2): 
ponsáveis por $90 \%$ de toda a literatura científica produzida na América Latina e Caribe na área de saúde.

No Brasil, a área de saúde pública em particular, é responsável por $60,7 \%$ de toda a produção científica. Entre os artigos publicados por autores residentes nos países em questão, nos periódicos internacionais importantes de epidemiologia ${ }^{3}, 66 \%$ foram de autores brasileiros, e toda a produção levantada no período, $61,7 \%$ concentrou-se no segundo período de estudo (1983-1992 versus 1973-1982), revelando o importante crescimento da pesquisa em toda a região.

Outro levantamento ${ }^{4}$, realizado a partir dos dados de avaliação da Capes das pósgraduações em Saúde Coletiva, registrou um total de 4.891 publicações científicas nesta área, no período de 1990 a 1995, revelando um crescimento da produção científica neste período. A medida da produção das pós-graduações é, sem dúvida, um importante indicador, uma vez que a maior parte da produção científica brasileira ocorre nesse âmbito.

Embora esse estudo não tenha discriminado a área , sabe-se que a epidemiologia tem um importante peso. Vale ressaltar que boa parte da produção analisada ocorreu em periódicos científicos ou livros publicados no país $(37,4 \%)$ e resumos em congressos nacionais (30,3\%). Dessa forma, abrese aqui a necessidade de uma discussão breve sobre os veículos de difusão de conhecimento científico na área de saúde coletiva no Brasil.

Da produção científica brasileira em epidemiologia publicada no período de 1995 a 1997, periódicos nacionais representaram $44,0 \%$, periódicos estrangeiros $26,9 \%$, livros completos, 6,3\% e capítulos de livros, 22,7\% (DGPB, 1997). As principais bases de dados internacionais, que incluem a área da epidemiologia, são o Medline e o Lilacs. O primeiro, embora tenha uma cobertura ampla, comparada a outras bases, confere uma atenção especial às revistas médicas da América do Norte ${ }^{5}$.

A produção científica brasileira pode e deve, cada vez mais, ser divulgada em revistas internacionais importantes e indexadas em bases de dados como o Medline. Entretanto, não se pode restringir a publicação a este tipo de periódico, uma vez que muitos trabalhos de relevância têm como público-alvo a comunidade nacional.

Dessa forma, periódicos científicos nacionais da área de saúde pública merecem ser prestigiados com o objetivo de obterem sua indexação em bases de dados da América Latina, hoje consagradas por sua qualidade e importância, como a Lilacs e, mais recentemente, o projeto SciELO. O grande objetivo destas bases/projetos é maximizar a disseminação da produção científica brasileira em diferentes áreas do conhecimento.

Um dos indicadores de aumento de produção sem o correspondente aumento de veículos de difusão é que revistas científicas nacionais mais importantes sofrem uma grande sobrecarga de trabalho. $\mathrm{O}$ intenso fluxo de material aos seus editores não é avaliado e divulgado em tempo adequado.

A incorporação de resultados de pesquisas pelos prestadores de serviços de saúde e a população em geral persiste como problema timidamente enfrentado. A transferência de conhecimentos não é um ato mecânico e raramente é incorporada rapidamente.

$\mathrm{O}$ conhecimento que se destina àqueles primeiros tem ocorrido por meio de uma importante colaboração entre a academia e os serviços, mostrando que as instituições de ensino e pesquisa podem auxiliar na solução dos problemas levantados nos serviços. A divulgação do conhecimento adqui-

\footnotetext{
${ }^{3}$ American Journal of Epidemiology, American Journal of Public Health, American Journal of Tropical Medicine and Hygiene, Bulletin of the World Health Organization, Epidemiology and Infection, International Journal of Epidemiology, Journal of Clinical Epidemiology, Medical Care, Preventive Medicine, Public Health Reports and Transaction of the Royal Society of Tropical Medicine and Hygiene

${ }^{4}$ Viacava F, Ramos CL. Difusão da produção científica dos cursos de pós-graduação em Saúde Coletiva. Ciência Saúde Coletiva 1997;2(1/2). ${ }^{5}$ Coimbra Jr CEA. Produção científica brasileira em saúde pública e as bases bibliográficas internacionais. Cad Saúde Pública 1999; 15: 883-8.
} 
rido em veículos adequados facilitaria sua utilização na prática dos serviços. Além disso, a população leiga pode ser alcançada se conhecimentos gerados através das pesquisas da área forem divulgados na imprensa voltada a esse público-alvo.

Os trabalhos científicos permitem verificar a diversidade de temas abordados, confirmando as observações dos planos anteriores. Nota-se a presença dos temas tradicionais, como as doenças transmissíveis (26,6\% das linhas de pesquisa registradas no DGPB) e saúde materno-infantil (19,5\%). A eles se incorporam os estudos sobre doenças crônicas $(13,4 \%)$ e serviços de saúde $(7,1 \%)$. Entretanto, temas importantes, embora presentes na agenda de pesquisa, ainda representam uma baixa proporção de atividade na área, como por exemplo, as causas externas $(2,8 \%)$ e a saúde mental $(3,3 \%)$.

Além das investigações temáticas, registram-se pesquisas de natureza metodológica e conceitual, incluindo pesquisa em epidemiologia, ciências sociais $(5,6 \%)$ e bioestatística (3,5\%). Uma aproximação da epidemiologia com as áreas básicas, tais como imunologia, genética, clínica e outras, como a geografia e a antropologia, também tem se constatado, permitindo uma maior interdisciplinaridade na abordagem dos objetos de estudo.

O aumento da produção de conhecimento em geral, de novos cursos e de doutores e pesquisadores no Brasil não foi acompanhado de um implemento de recursos injetados pelos governos federal e estaduais nessas áreas. Ao contrário do que se esperava, houve uma redução nos recursos destinados à ciência, à tecnologia e às universidades públicas brasileiras, com exceção do Estado de São Paulo. Nota-se, ainda, uma importante defasagem entre o número de bolsas destinadas a pesquisadores e estudantes e o dinamismo da área, além da relativa perda do poder aquisitivo das bolsas concedidas. Grupos já consolidados, assim como aqueles emergentes, também não estão recebendo financiamento que atenda a suas necessidades e especificidades.
As fundações de pesquisa estaduais, salvo exceções como a Fapesp, nem sempre cumprem seu papel e, muitas delas, não destinam ou fazem uso político das verbas que deveriam à ciência e à tecnologia, de acordo com as constituições estaduais. Além disso, essas fundações não são sensibilizadas para as necessidades específicas da área de saúde coletiva.

Ao mesmo tempo, observa-se um incremento do financiamento de pesquisa pelos serviços de saúde. Esse fato reflete uma maior integração serviço-academia que é benéfica para ambas as partes. Um risco dessa forma de contratação é deixar de compatibilizar a autonomia do pesquisador com as necessidades da instituição interessada. Essa forma de financiamento não deve, em nenhum momento, impedir que pesquisas não vinculadas aos serviços continuem a receber financiamento de outras fontes.

Observa-se, nesse momento, a criação de vários fundos visando o financiamento da pesquisa científica e tecnológica. Os fundos setoriais recém-aprovados e os que ainda estão em constituição - desde que não impliquem em cortes nos recursos tradicionais à disposição das instituições e pesquisadores - alimentam expectativas de novos recursos para o setor.

É motivo de preocupação para os epidemiologistas a não participação da comunidade científica nos debates sobre a constituição da agência nacional de fomento à pesquisa na área da saúde, ora em tramitação no Ministério da Saúde e no Congresso Nacional. Essa circunstância pode pôr a perder os aspectos positivos eventualmente incluídos na proposta de uma agência dessa natureza, pois representa a exclusão daqueles que serão seus usuários.

A esse cenário positivo, dando uma idéia dos avanços alcançados pela epidemiologia, devem-se, entretanto, acrescentar problemas que impõem obstáculos ao pleno crescimento da disciplina. A ausência de políticas explícitas de desenvolvimento científico e tecnológico em saúde afeta a orientação e o financiamento da pesquisa, 
constituindo-se em entrave para a consolidação dos avanços já alcançados. Isso leva à dificuldade de expansão da formação do quadro de pesquisadores, impedindo, a curto prazo, mecanismos de inserção deste nos espaços próprios para seu crescimento e para garantir conseqüência à sua capacidade de trabalho.

Apesar de ser quase consensual que a epidemiologia deve centrar-se no estudo da distribuição e dos determinantes de estados e eventos relacionados à saúde em populações, com vistas à prevenção ou controle, existe um razoável grau de desacordo entre epidemiologistas com relação às bases conceituais e metodológicas para abordar tais questões. A própria complexidade do fenômeno saúde-doença, que torna possível enfocá-lo desde os seus determinantes macro-sociais aos seus mecanismos micromoleculares, fundamenta a dificuldade na formação dos consensos necessários.

Um outro aspecto sobre o qual se observam divergências entre os epidemiologistas é a própria aplicabilidade do conhecimento epidemiológico. Nos extremos, enquanto alguns privilegiam o potencial da disciplina para os estudos causais fundamentais, outros enfatizam sua aplicação imediata em questões como o fortalecimento dos serviços de saúde. Vistos positivamente, esses antagonismos constituem estímulos para o desenvolvimento conceitual e metodológico da disciplina e estão na base da sua estimulante diversidade.

Tendo em vista as características deste plano, buscou-se um eixo de organização das prioridades científicas da disciplina que, respeitando a diversidade das linhas de investigação epidemiológica em curso no país, permitisse estimular alguns elementos unificadores e de comunicação entre elas. Tais eixos temáticos, para serem aceitáveis pela comunidade de epidemiologistas, devem estar em consonância com as necessidades de novos conhecimentos que não somente contribuam para a solução dos grandes problemas de saúde da população brasileira, mas também permitam que sejam compartilhadas as grandes questões universais referentes à saúde da população humana. Nesta perspectiva três eixos foram identificados:

- Desigualdade em saúde. Tema tradicional e consistente nas mais diversas linhas da investigação epidemiológica, desde quando praticamente todos os eventos epidemiológicos apresentam padrões que se caracterizam pela sua distribuição desigual entre os vários grupos sociais. A sua importância cresce com a ampliação ou, pelo menos, a não redução das desigualdades nas sociedades ocidentais e, em especial, na sociedade brasileira, mesmo quando eventualmente ocorrem melhorias globais nos níveis de vida.

A complexificação das organizações sociais modernas aumentou as possibilidades de expressão das desigualdades em saúde, que se apresentam nas mais diversas dimensões (classes sociais, grupos étnicos e culturais, gêneros etc.). Está claro que a dinâmica dos fenômenos epidemiológicos está em grande parte definida pela conformação que assumem tais desigualdades. Há evidente necessidade de se ampliar a documentação de seus efeitos na saúde e o entendimento de seus mecanismos causais, objetivando a construção de práticas de saúde orientadas para a busca da eqüidade.

- Ambiente e qualidade de vida. A epidemiologia tem sua origem associada à compreensão da importância do ambiente na saúde e aos movimentos voltados às transformações ambientais que tivessem impacto sobre a saúde humana. Nos países subdesenvolvidos, os problemas ambientais tradicionais responsáveis pela ocorrência de altas taxas de doenças infecciosas se associam a um conjunto novo de problemas decorrentes do processo crescente e pouco regulamentado de introdução de processos tecnológicos com alto impacto sobre o ambiente e, muitas vezes, sobre a saúde.

Cada vez fica mais clara a importância dos fatores ambientais, não somente sobre a qua- 
lidade de vida na atualidade, mas também, a longo prazo, na própria sobrevivência da espécie humana. Grande parte das investigações epidemiológicas envolve dimensões ambientais no seu sentido lato. Portanto, recolocar esses conhecimentos no centro dos debates sobre as perspectivas da vida e da saúde humana é uma obrigação e um compromisso histórico da epidemiologia.

- Avaliação do impacto das tecnologias nos níveis de saúde. A vida nas sociedades modernas é caracterizada pela presença crescente de novas tecnologias, muitas delas supostamente direcionadas para produzir benefícios à saúde. Com freqüência, muitas dessas tecnologias têm sido criticadas por não produzirem efeitos ou mesmo por seus possíveis efeitos negativos sobre a saúde. Alguns paradigmas contemporâneos maximizam o papel de tais tecnologias e tentam inclusive nos impor a idéia de que as melhorias nas condições de saúde são, em grande medida, resultante da sua incorporação ao cotidiano da vida das sociedades e, portanto, separadas dos contextos sociais, econômicos e políticos. Tudo isso no sentido de aumentar as expectativas de utilização e justificar os altos e crescentes custos que acarreta.

A epidemiologia dispõe de vários recursos investigativos para desempenhar a função de desenvolver métodos avaliativos e produzir informações que permitam separar criteriosamente as tecnologias apropriadas daquelas inúteis ou perigosas.

\section{Política de Desenvolvimento Científico e Tecnológico e Financiamento à Pesquisa}

\section{Problemas}

- Ausência de políticas consistentes com o adequado e regular desenvolvimento científico e tecnológico em saúde de médio e longo prazo.

- Participação limitada da comunidade científica da área nas agências e foros decisórios sobre as políticas.
- Destinação de recursos financeiros não acompanhada do crescimento dos grupos de pesquisa na área.

- Instabilidade no financiamento científico, acarretando a perda de credibilidade nas agências de fomento e levando grupos de excelência, assim como os emergentes, a não receberem financiamentos adequados.

- Falta de cumprimento da maioria das FAP's dos preceitos das constituições estaduais, que prevêem recursos do estado para o financiamento da pesquisa.

\section{Proposições}

- Implementação de uma política coerente que permita o adequado desenvolvimento científico e tecnológico da área levando em conta a realidade de saúde do país.

- Ampliação dos canais efetivos de participação organizada da comunidade científica nos órgãos competentes da área.

- Ampliação dos recursos para a área de saúde coletiva, acompanhando o seu crescimento.

- Garantia de continuidade e regularidade de financiamento adequado, a longo e médio prazos, aos grupos consolidados e aos emergentes, por intermédio de canais diferenciados de alocação de recursos.

- Ação coordenada com a SBPC, pressionando os governos onde existem FAP's, para o cumprimento da constituição. Sensibilização das FAP's em relação às necessidades específicas da área da Saúde Coletiva.

\section{Divulgação da Produção de Conhecimentos}

\section{Problemas}

- Número reduzido de periódicos indexados nacionais que dêem conta da divulgação da crescente produção científica da área no país.

- Espaços reduzidos de divulgação das pesquisas voltadas ao serviço. 
- Dificuldade de acesso à produção científica internacional pela comunidade científica nacional.

- Escassez de divulgação dos produtos das investigações epidemiológicas para o público em geral.

\section{Proposições}

- Fortalecimento da Revista de Epidemiologia da Abrasco, visando a sua indexação e transformação em um órgão de divulgação da produção científica brasileira na área de epidemiologia. Produção da Revista on-line para divulgação via Internet.

- Fortalecimento e adequação do Informe Epidemiológico do SUS no papel de divulgação de pesquisas e estudos voltados aos serviços de saúde.

- Criação de mecanismos que permitam a compilação e divulgação da produção científica de brasileiros em nível internacional.

- Acompanhamento dos esforços desenvolvidos no Brasil e no exterior, no sentido de franquia de acesso à produção científica internacional via Internet.

- Estímulo à divulgação da produção científica nacional na área em veículos como os órgãos da SBPC (Jornal da Ciência e Revista Ciência Hoje) e nos espaços destinados à saúde, à ciência $\mathrm{e}$ tecnologia e ao meio ambiente nos grandes jornais.

- Estímulo ao posicionamento da comunidade científica da epidemiologia frente a temas e problemas de interesse nacional.

\section{Questões Gerais}

\section{Problemas}

- Restrito intercâmbio entre as instituições nacionais, pesquisadores e alunos de pós-graduação.

- Frágil e lenta articulação entre a epidemiologia e o planejamento em saúde.

\section{Proposições}

- Criação de mecanismos capazes de implementar o intercâmbio entre instituições e profissionais para a aquisição de novas técnicas, incluindo cursos à distância e emprego de vídeoconferências.

- Elaboração de um Banco de Dados das disciplinas de Pós-graduação em Saúde Coletiva, e respectivas ementas, cujo acesso se dê através de links da Abrasco com as instituições de ensino e pesquisa.

- Organização de um Seminário organizado pela Abrasco sobre o uso da epidemiologia na avaliação dos serviços de saúde.

\section{A Epidemiologia nas Políticas, Programas e Serviços de Saúde}

As questões analisadas pelo II Plano Diretor continuam atuais, bem como muitos dos problemas apontados continuam a merecer uma atenção especial no sentido da busca de soluções. No entanto, são também incontáveis os avanços ocorridos muitos deles já apontados pelo II Plano, quando comparado com o anterior - atestando principalmente o expressivo desenvolvimento da Epidemiologia no Brasil, seja na formação de recursos humanos, na pesquisa, seja nos serviços de saúde.

A consolidação da área pode ser avaliada pela densidade crescente dos congressos de epidemiologia , pelos cursos de especialização desenvolvidos em várias regiões do país, pelo número de epidemiologistas completando sua formação, tanto no país quanto no exterior, bem como pela crescente produção científica. Essa consolidação da área também se refletiu nos serviços de saúde, ainda que de forma tímida e aquém do possível e do desejável.

O processo de descentralização e municipalização dos serviços de saúde tem representado novos espaços para o desenvolvimento da área de epidemiologia em serviços. Mais recentemente, a partir da edição das 
normas operacionais de 1993 e de 1996, conta-se com novas condições de gestão no âmbito da administração municipal em saúde, com a redefinição dos papéis do nível estadual de gestão do SUS, com modificações organizacionais e com a implementação de políticas de financiamento de serviços no âmbito do Ministério da Saúde.

Algumas modificações importantes ocorreram no nível estadual de gestão do SUS. Os setores de vigilância epidemiológica e sanitária e a área de informação em saúde, que tradicionalmente desempenhavam as tarefas de atuação direta em prevenção e controle de riscos e agravos, em produção, consolidação e análise de dados de interesse em saúde desagregados para o nível local, redefinem suas atribuições e passam a exercer novos papéis relacionados à normatização e regulamentação, capacitação de pessoal e programação regional e estadual de ações e serviços, coordenando o pacto entre gestores para sua implantação.

Dessa forma, também se modificam os padrões de utilização da informação epidemiológica no nível estadual de gestão do SUS, passando esta a exercer de forma crescente o papel de suporte à decisão em planejamento, para o estabelecimento de prioridades e para a formulação e implementação de políticas de saúde.

A essas iniciativas se somaram experiências inovadoras da aplicação do conhecimento epidemiológico na reorganização da oferta de serviços e da rede de unidades, inclusive, em alguns municípios, também no desenvolvimento de sistemas locais de saúde que têm como unidade operacional os distritos sanitários.

Em decorrência desses processos, práticas relativas à aplicação da epidemiologia aos serviços, antes realizadas por profissionais dos níveis regional e central das secretarias estaduais de saúde, foram incorporadas às atividades e tarefas dos grupos profissionais de atuação municipal.

Além disso, as exigências do registro de dados e de alimentação dos sistemas de informação em saúde, tanto os relativos à pro- dução de serviços ambulatoriais e hospitalares do SUS quanto os da área de vigilância epidemiológica, para o cumprimento dos requisitos da descentralização, têm oferecido novas oportunidades para o crescimento desta área nas secretarias municipais de saúde.

Entre os avanços ocorridos no período referente aos dois Planos Diretores, merece destaque a área de informações epidemiológicas com a maior disponibilidade de dados, principalmente via Internet.

No âmbito dos sistemas de informações para o registro de dados da assistência ambulatorial e hospitalar, as modificações introduzidas nas tabelas de procedimentos, a implantação da 10a Revisão da CID e a ampliação da cobertura de informatização desses sistemas nos níveis estadual e municipal têm propiciado o aumento da sua utilização como fonte de dados para o estudo e a caracterização de situações de saúde e para o acompanhamento e a avaliação de serviços e intervenções.

O grande avanço com relação a essa questão foi talvez a maior facilidade de acesso aos grandes bancos de dados, inclusive por meios eletrônicos. Essa área de informação em saúde, não somente informação epidemiológica, tem obtido avanços importantes .

Iniciativas têm sido implementadas em conjunto pelo Ministério da Saúde, Opas, Abrasco e Universidades, das quais se destacam a RNIS (Rede Nacional de Informações em Saúde), a Ripsa (Rede Interagencial de Informações para a Saúde), a implantação do IVR (Índice de Valorização de Resultados) e a intensificação das atividades de capacitação de profissionais para as áreas de informação e informática em saúde podem modificar em prazo relativamente curto o panorama habitual de baixa utilização dessas fontes de dados e promover maior integração entre as áreas de vigilância em saúde e de assistência e gerência de rede de unidades, tradicionalmente distintas e desarticuladas nas instâncias de gestão do SUS.

Com relação à realização de inquéritos, devem ser ressaltados a PNAD-SAÚDE 98, 
que se centrou na utilização de serviços de saúde; Pesquisa Nacional de Saúde da Mulher e da Criança, promovido pela Benfam/ DST-AIDS; Inquérito Epidemiológico de Doença de Chagas, realizado pelo Instituto Brasileiro de Medicina Tropical; Análise da Cobertura Vacinal de Febre Amarela na Amazônia Legal; Elegibilidade da água de consumo humano por meio de programa apoiado pela OPS e CDC. Avançou-se, no entanto, muito pouco na realização de inquéritos de saúde de base populacional nos diferentes âmbitos - estadual, regional e federal.

Com relação às discussões metodológicas, relativas à abordagem das desigualdades, ressalta-se o seminário realizado pela Abrasco sobre Condições de Vida e Saúde, em que foram discutidos diversos aspectos referentes a essa questão. Também foi realizada uma oficina de trabalho sobre o tema no IV Congresso de Epidemiologia, cujos resultados ainda não foram divulgados. A Abrasco publicou uma coletânea - Desigualdade em Saúde - compilada a partir de palestras realizadas no III Congresso de Epidemiologia.

Muita coisa ainda precisa ser feita e são muitas as dificuldades operacionais para avaliar as desigualdades em saúde. A produção de conhecimento sobre o tema, em nosso meio, é muito escassa frente à dimensão do problema e, até o momento, o Cenepi pouco avançou nessa perspectiva.

Nos níveis descentralizados, observaram-se diversos avanços tais como a utilização de informações para diagnóstico local de saúde, tanto pela criação de sistemas locais de mortalidade quanto pelo desenvolvimento e aplicação de metodologias apropriadas, como as desenvolvidas nos programas assessorados pela Cooperação Italiana, pelas propostas dos Silos, Territorialização e outras.

Cresceram também os programas de vigilância de óbitos infantis vinculados a programas de 'Enfoque de Risco', que exemplificam bem a interface entre conceitos e métodos da epidemiologia e programas e administração dos serviços de saúde.
A área de doenças não transmissíveis e os problemas de saúde na terceira idade merecem atenção crescente dos serviços de saúde, com incorporação de técnicos e desenvolvimento de programas.

Emergem estudos epidemiológicos de saúde bucal, visando a subsidiar e avaliar programas, particularmente o de fluoretação da água de abastecimento. Surgiram também os primeiros estudos, em nosso meio, que avaliam a poluição do ar e os riscos à saúde, subsidiando medidas de controle ou pelo menos formas de atenuar os riscos, como a monitorização da qualidade do ar e o rodízio de veículos.

$\mathrm{Na}$ área da vigilância à saúde, grande avanço talvez ocorra com o Sistema de Vigilância Epidemiológica e Ambiental para o Sistema Único de Saúde (Vigisus), que o Ministério da Saúde vem implementando. Esse empreendimento incorpora questões amplas referentes à reestruturação dos serviços, equipamentos, formação de recursos humanos e pesquisas, contando com substancial financiamento, inclusive do Banco Mundial. A maior flexibilidade de utilização dos recursos dessa proposta deverá imprimir maior agilidade executiva às secretarias estaduais e municipais e ampliar a articulação entre os setores de produção científica e acadêmica e a rede de serviços de saúde.

A experiência vivida em eventos inusitados, como o de Caruaru, demonstrou avanço, no sentido de reconhecer que os serviços de epidemiologia, em articulação com serviços acadêmicos e com centros de pesquisa, podem responder de forma mais efetiva aos problemas emergentes.

Apesar da área da avaliação vir sendo identificada como de grande importância, o seu desenvolvimento ainda é tímido, principalmente quando se refere à interface entre epidemiologia e serviços de saúde.

O maior desenvolvimento vem sendo realizado pelas disciplinas de administração e planejamento. Contudo, têm sido oferecidas disciplinas e cursos de avaliação de serviços de saúde nas universidade e publicados artigos específicos sobre essa ques- 
tão, inclusive com número temático sobre a 'epidemiologia na avaliação dos serviços de saúde'.

Do ponto de vista institucional, constata-se que é de fundamental importância a existência de um Órgão Central de Epidemiologia para o SUS, tendo em vista a permanente necessidade de equacionamento dos complexos problemas de saúde da população brasileira.

No entanto, as atribuições do Cenepi não vêm sendo executadas a contento, devido à insuficiência de recursos e às dificuldades decorrentes de sua inserção institucional.

Reconhece-se, assim, ser urgente a necessidade de fortalecer o Órgão Central de Epidemiologia do SUS, com a mobilização da competência técnico-científica no campo da epidemiologia no país.

Não foi possível identificar ações desenvolvidas no sentido das propostas constantes no II Plano com relação ao "fortalecimento da articulação entre os setores de Planejamento, Administração e Epidemiologia nos Serviços de Saúde" . Assim, é fundamental a conclusão do processo em curso de descentralização da ECD (Portaria 1399/99). Certamente, essa articulação vem ocorrendo de várias maneiras sem, contudo, haver uma sistematização e uma divulgação de experiências concretas que pudessem estimular sua ampliação.

A criação de conselhos de saúde nos níveis estadual e municipal de gestão do SUS e o desenvolvimento do exercício dos seus papéis de deliberação sobre diretrizes setoriais, de acompanhamento e de avaliação da gestão, têm ampliado o uso das informações epidemiológicas, exigindo dos serviços a preparação e a análise de informações que subsidiem esses processos. Isso se reflete, também, no desenvolvimento da área de epidemiologia em serviços.

Aspectos políticos das relações entre governo e sociedade, definidos como controle social do SUS e representados por esses conselhos de saúde, influem de forma crescente na análise de situações. Nessa vertente, as contribuições metodológicas e práticas da epidemiologia são evidentes.

Apesar dos avanços acima referidos, a transição vivida pelos serviços públicos de saúde, e principalmente a crise econômica, vêm levando a um esvaziamento do setor, fazendo com que, de uma forma contraditória, a consolidação da área de epidemiologia tenha um impacto menor do que o esperado nos serviços.

Esses problemas já haviam sido mencionados no II Plano Diretor, que fez referência à "descontinuidade dos investimentos nos serviços de saúde e a falta de uma política adequada de recursos humanos" levando "a uma baixa fixação, ou mesmo, à evasão de profissionais capacitados".

Várias questões ligadas à epidemiologia nos serviços de saúde ainda merecem reflexão e uma ampla discussão na busca de propostas viáveis. O II Plano Diretor identificou três áreas da prática epidemiológica voltadas para a atuação dos serviços de saúde: estudo da situação de saúde; vigilância de doenças e agravos à saúde e avaliação dos serviços de saúde.

Constatando-se, no entanto, o avanço do desenvolvimento da epidemiologia nos últimos anos, seja nas formulações/normatizações da política nacional de saúde, seja nas práticas conduzidas pelos diferentes programas no âmbito do sistema de saúde, especialmente nos municípios, considerouse que a divisão nas áreas utilizadas no II Plano Diretor não contemplaria a complexidade dos questionamentos atuais.

Dessa forma, foi proposto o seguinte agrupamento de áreas relativas à epidemiologia nos programas, políticas e serviços de saúde:

- Sistemas de Informação de Interesse Epidemiológico

- Desenvolvimento e Utilização de Metodologias para Análise das Situações e das Intervenções em Saúde

- Práticas Epidemiológicas nos Programas e Serviços

- Políticas de Recursos Humanos

${ }^{6}$ II Plano Diretor para o Desenvolvimento da epidemiologia no Brasil, 1995-1999. Rio de Janeiro: Abrasco, 1995. 
- Vigilância em Saúde

- Avaliação em Saúde

- Inserção nas Políticas Intra e Intersetoriais

\section{Sistemas de Informação de Interesse} Epidemiológico

\section{Problemas}

- Mecanismos coercitivos associando fluxos de informações ao repasse de recursos, comprometendo a adequada utilização e o controle de qualidade do sistema.

- Baixa qualificação e ausência de supervisão na operação dos sistemas de informação epidemiológica.

- Predomínio, na gestão dos sistemas de informação, da lógica centralizada e de processamento dos dados em detrimento de sua análise e das necessidades dos níveis locais do sistema de saúde, em especial no desencadeamento de ações imediatas.

- Dificuldade de acesso às informações geradas pelos sistemas, tanto pelas equipes de saúde quanto pelos gestores ou a sociedade em geral.

- Oferta de informações trabalhadas em linguagem inacessível aos usuários (equipes, conselhos de saúde, movimento social organizado etc.), perdendo sua potencialidade de transformação das práticas e da política de saúde.

- Baixo envolvimento do Cenepi na construção dos sistemas de informação em saúde que contemple agravos específicos, duplicando os sistemas existentes (RCBP, Registro Hospitalar, Siscolo, Silab, Siab, Sisprenatal, procedimentos de alta e média complexidade etc.).

- Inadequação e/ou incompatibilidade dos sistemas de informação em saúde para sua utilização articulada e com interesse epidemiológico.

- Falta de regulamentação e acesso às informações geradas fora dos SUS, como por exemplo, os dados do sistema de Saúde Suplementar de internações hospitalares de pacientes particulares e de clientes de planos e seguros de saúde ou das informações geradas em outros setores, como segurança pública, trânsito, habitação, entre outras.

\section{Proposições}

- Estabelecer mecanismos de controle de qualidade de informações no SUS, privilegiando aquelas relacionadas à pactuação de metas e/ou incentivos locais.

- Ampliar e garantir o treinamento do pessoal responsável pelo processamento dos Sistemas de Informações Epidemiológicas.

- Estimular a realização de estudos de validade e confiabilidade das informações dos sistemas de interesse epidemiológico nacional.

- Substituir a ênfase do enfoque processual pelo analítico nos Sistemas de Informações Epidemiológicas, de forma a atender oportunamente as necessidades de acesso dos níveis local e regional ao registro de ocorrências que exijam intervenções imediatas.

- Estimular e capacitar equipes de saúde para produzir, com os softwares existentes e de domínio público, tabulações e análises de dados com ênfase nas informações adequadas ao desencadeamento de intervenções e sua avaliação.

- Democratizar o acesso às informações epidemiológicas, tanto brutas quanto trabalhadas, resguardando a confidencialidade de informações individuais sem prejuízo da necessidade de intervenção coletiva de benefício público.

- Dar visibilidade aos problemas de saúde mais relevantes, por meio do repasse para a sociedade de informações epidemiológicas, incluindo as repercussões dos riscos atuais e potenciais para o estado de saúde da população em cada situação concreta, utilizando os meios de comunicação de massa e os órgãos de controle social.

- Cuidar para que as informações repassadas aos usuários dos Sistemas de Informações Epidemiológicas sejam inteligíveis e estimulem o uso para a ação. 
- Reclamar maior envolvimento do Cenepi com as Secretaria de Assistência a Saúde e a Secretaria de Políticas, do Ministério da Saúde, para adequar os sistemas de informação em saúde já existentes, evitando sua multiplicação e fragmentação.

- Acompanhar e agilizar o processo de compatibilização e homogeneização de variáveis dos diferentes sistemas para uso epidemiológico, incluindo os níveis de desagregação de dados dos sistemas locais, como SIA/Siab.

- Discutir com a ANS formas de se estabelecer os limites de acesso e utilização dos dados disponíveis sobre os clientes dos planos de seguros de saúde em estudos epidemiológicos, estabelecendo critérios para garantir a confidencialidade desses dados.

- Buscar estabelecer fluxos de repasse dos dados de interesse do sistema de saúde com outros setores, tais como segurança pública e trânsito, tanto para a melhoria de qualidade dos sistemas de informação epidemiológica quanto para uso em ações intersetoriais.

\section{Desenvolvimento e Utilização de Metodologias Apropriadas para Análise das Situações e das Intervenções em Saúde}

\section{Problemas}

- Defasagem entre os avanços conceituais e abordagens epidemiológicas desenvolvidas nas universidades, relativas ao complexo saúde-doença-intervenção e o reducionismo das investigações e aplicações de normas descontextualizadas nas práticas epidemiológicas.

- Descontinuidade técnico-financeira e excessiva informalidade nas relações estabelecidas entre instituições acadêmicas e os serviços, comprometendo muitas vezes a própria rotina operacional.

- Insuficiência na capacitação, em métodos e técnicas de análise epidemiológica para os profissionais dos serviços de saúde.
- Carência da oferta de metodologias de fácil manejo operacional para utilização, pelos diversos níveis do SUS, em especial aquelas adequadas ao nível local. - Pouca utilização do geoprocessamento como instrumento de trabalho nos serviços de saúde.

- Inquéritos de base populacional insuficientes e, quando realizados, restritos aos interesses do nível central, impossibilitando seu uso para atuação regional e local.

- Baixa utilização de estudos sobre desigualdades em saúde no estabelecimento de prioridades para as ações programáticas.

\section{Proposições}

- Estabelecimento de parceria continuada das instituições de ensino-pesquisa com os serviços, com base em: contratualização, definindo claramente atribuições mútuas, fontes de financiamentos e produtos esperados; utilização de abordagens educacionais e de pesquisa interdisciplinares, contextualizadas, consensuadas e orientadas pela oportuna tomada de decisões, com recíproca oportunidade de aprendizagem e produção do conhecimento; democratização das informações geradas por esses produtos e compromisso mútuo com a implementação das recomendações formuladas.

- Necessidade de se estabelecer política de fomento e financiamento de pesquisas operacionais para responder as questões oriundas dos serviços de saúde.

- Enfatizar a necessidade de programas específicos, nas agências financiadoras nacionais e estaduais, que fomentem a pesquisa epidemiológica nos sistemas e serviços de saúde.

- Definir conteúdos mínimos de capacitação, revalorizando a epidemiologia descritiva.

- Desenvolver metodologias simplificadas, com o requerido rigor científico, para atender as necessidades de informações 
inexistentes nos bancos de dados atuais.

- Implementar a produção e disseminação dos métodos de análise temporal, espacial e de 'pequenos números', bem como sua disponibilização em softwares de domínio público.

- Promover, em parceria com o IBGE, o acesso facilitado aos dados dos setores censitários devidamente compatibilizados aos sistemas de informação epidemiológica (SIM, Sinan e Sinasc) e às tecnologias do geoprocessamanto.

- Ampliar a capacidade técnico-científica na área da saúde para formulação e realização de inquéritos adequados aos três níveis do sistema (municipal, estadual e federal).

- Estimular a incorporação dos resultados de estudos sobre desigualdades em saúde para o planejamento e avaliação dos efeitos das intervenções.

\section{Práticas Epidemiológicas nos}

Programas e Serviços de Saúde

\section{Políticas de Recursos Humanos}

\section{Problemas}

- Inexistência de uma carreira para o profissional de epidemiologia.

- Salários não atraentes.

- Falta de concursos para absorção das pessoas formadas.

- Grande rotatividade de pessoal, resultando em perda do pessoal treinado.

- Abordagens de capacitação e reciclagem descoladas do processo de trabalho.

- Distanciamento na relação com os gestores.

\section{Proposições}

- Atuação da Abrasco junto ao Cenepi, ao Conass e ao Conasems para advogar a necessidade de estabelecer Plano de Carreira.

- Prever forma de financiamento para a remuneração dos profissionais da área (a exemplo do PSF), sem o que a definição da carreira não terá efeito prático.
- Absorver, por meio de concurso público, pessoal treinado.

- Estabelecer vinculação permanente entre os serviços e as instituições de ensino-pesquisa em epidemiologia que garantam o ensino e o acompanhamento continuados.

- Desenvolver cursos na rotina de trabalho, com levantamentos de problemas concretos de cada área da clientela e definição de conteúdos específicos.

- Realizar oficinas para solução de problemas específicos, incluindo análise dos processos internos de trabalho dos serviços, visando ao fortalecimento da epidemiologia nos níveis locais.

- Incluir, nos cursos de capacitação de gestores, conteúdos que evidenciem a importância e potencialidade do instrumental epidemiológico para o processo decisório, sensibilizando-os quanto à necessidade de apoio à área.

- Desenvolver cursos de gestores de vigilância epidemiológica.

- Incluir 'epidemiologia em serviços' na formação de recursos humanos dos cursos de epidemiologia stricto sensu.

- Estabelecer mecanismos para garantir a qualidade da capacitação oferecida, incorporando, nas propostas de capacitação, mecanismos de avaliação para a atualização permanente dos conteúdos (lacunas, erros, acertos) e o acompanhamento dos egressos.

\section{Vigilância em Saúde}

\section{Problemas}

- Vigilância epidemiológica centrada em agravos de notificação compulsória, dissociada da vigilância sanitária, das redes assistencial e laboratorial.

- Baixa institucionalização e estrutura inadequada dos núcleos de epidemiologia das unidades hospitalares.

- Inexistência de diretrizes com critérios para certificação de estados e municípios na gestão e avaliação de qualidade dos sistemas de vigilância epidemiológica. 
Proposições

- Definir mecanismos e incentivos financeiros nos estados e municípios para que implantem projetos inovadores, ampliando o escopo da vigilância dos agravos em saúde, integrando neste processo outras áreas do setor como a assistência, a vigilância sanitária, a saúde do trabalhador, além da articulação inter-setorial na formulação de políticas públicas que, sabidamente, interferem no processo saúde-doença.

- Desenvolver sistema de vigilância para doenças emergentes.

- Estruturar a Vigilância Ambiental, integrada à Vigilância Epidemiológica, resguardando as abordagens diferenciadas de acordo com a complexidade dos múltiplos determinantes e ações de controle necessárias.

- Integrar a rede laboratorial nas diferentes etapas de implementação do Sistema de Vigilância (incluindo prioridades da alocação de recursos e qualificação).

- Organização do fluxo de informações e amostras, respeitando-se as competências legais estabelecidas entre os diferentes níveis, com coordenação do Cenepi/Colab.

- Inclusão, sempre que pertinente, de técnicos de outras áreas em treinamento em vigilância epidemiológica (CBVE).

- Fortalecimento da estrutura dos núcleos de epidemiologia com a qualificação de pessoal e de equipamentos adequados para a análise de dados nas unidades hospitalares.

- Definição de critérios mínimos na estruturação e qualificação de equipes para gestão do sistema de vigilância epidemiológica.

\section{Avaliação em Saúde}

\section{Problemas}

- Baixa utilização de abordagens epidemiológicas nos estudos de monitora- mento e avaliação sobre a adequada implementação das ações de saúde.

- Insuficiência das avaliações de efetividade e impacto epidemiológico que, quando realizadas, freqüentemente não contemplam a análise das intervenções.

\section{Proposições}

- Inserir conteúdos relativos ao monitoramento e à avaliação das ações programáticas e serviços nos cursos promovidos pela Rede Nacional de Formação para o SNVE.

- Fomentar as pesquisas relativas à resolutividade dos programas e serviços de saúde.

- Considerar o monitoramento dos processos de assistência à saúde como etapa imprescindível para a avaliação dos serviços.

- Realizar, sempre que pertinente, análise das intervenções, correlacionando-a aos indicadores de impacto e eficiência.

- Promover reflexões sobre questões relativas aos custos da assistência frente àquelas do direito da atenção à saúde .

\section{Inserção nas Políticas Intra e Inter-setoriais}

\section{Problemas}

- Necessidade de maior inserção do Cenepi no SUS, com estabelecimento claro de suas características, competências e atribuições.

- Desarticulação das diversas áreas da saúde e outros setores na formulação e operacionalização das políticas nacionais, estaduais e municipais de saúde.

- Ações de epidemiologia circunscritas ao seu próprio âmbito, desintegradas das decisões gerenciais do setor saúde.

- Pouca participação do Cenepi nas formulações, implantação e /ou implementação de projetos nacionais de inegável importância epidemiológica, como o do Cartão SUS. 
Proposições

- Realizar gestões junto ao Governo Federal, visando a redefinir a inserção do Órgão Central de Epidemiologia do SUS na estrutura do Ministério da Saúde. Este novo órgão deverá ter autonomia administrativa; flexibilidade de gestão com subordinação aos mecanismos de controle social do Ministério da Saúde e carreira própria com remuneração adequada ao nível de competência exigida para seu quadro de recursos humanos.

- Solicitar a inserção, nesta nova estrutura, de um Comitê Consultivo com membros da comunidade acadêmica da rede de serviços de saúde e da Abrasco, com o objetivo de garantir a permanente participação na definição de suas diretrizes e políticas.

- Como definido em reuniões da comunidade e formalizado em documentos oficiais, esse órgão deveria efetivamente assumir as atribuições para as quais foi criado (incluindo o estabelecimento das condições para que estas ocorram, nos níveis descentralizados do sistema de saúde, quando pertinente):

- Análise da situação de saúde da população para subsidiar a formulação e implementação de políticas.

- Vigilância e controle de doenças e agravos à saúde, visando à redução da morbi-mortalidade.

- Avaliação do impacto das ações e programas de saúde para subsidiar a organização e gestão dos serviços de saúde.

- Desenvolvimento de recursos humanos na área.

- Fomento à pesquisa estratégica em epidemiologia.

- Manutenção e aprimoramento dos sistemas nacionais de informações de base epidemiológica.

- Disseminação das informações para o sistema e a sociedade.

- Articular ações da Abrasco/Cenepi junto ao Conass e Conasems, buscando apoio para o reconhecimento da área de vigilância à saúde como atividade própria e exclusiva do Estado.
- Ações da Abrasco/Cenepi junto ao Conass e Conasems, buscando valorizar as informações epidemiológicas como instrumental importante na definição de ações e intervenções prioritárias em saúde dentro de modelos técnicoassistenciais e de promoção à saúde, como o PSF, Cidade Saudável e outros modelos implementados no país.

- Implementar a articulação do Cenepi nos projetos das redes de informação e Cartão SUS.

- Participar da definição de critérios dos repasses do $\mathrm{PAB}$ que devem ser dirigidos para a saúde pública, acompanhando sua utilização e ampliando o campo de influências em todos os níveis de gestão (incluindo as Comissões Intergestoras), do nacional ao local .

- Integrar a pactuação dos indicadores e a programação dos recursos do Piso de Atenção Básica, com os indicadores e o teto financeiro da epidemiologia e do controle de doenças.

- Estimular, no nível estadual e, em especial, no nível municipal, a integração da epidemiologia com as diversas áreas técnicas e os diversos níveis gerenciais intra e inter-setoriais, no sentido de definir, conjuntamente, prioridades e intervenções em saúde.

- Ampliar a atuação da epidemiologia na assistência à saúde, atualmente restrita à rede básica e aos hospitais de doenças infecciosas, lembrando que os serviços de emergência e os hospitais de uma forma geral são espaços privilegiados de ocorrência de eventos que devem ser objeto de atenção da epidemiologia e da vigilância epidemiológica.

- Inserir na agenda do Conass e Conasems a discussão do III Plano Diretor de Epidemiologia, reiterando a idéia dessas instâncias se fazerem representar na Comissão de Epidemiologia da Abrasco.

Finalizando, vale ressaltar que o país tem uma produção científica e de práticas epidemiológicas rica, criativa e plural, que 
abrange os campos teórico, metodológico e aplicado, em diversas áreas específicas, como: doenças crônico-degenerativas, acidentes, ambiente, trabalho, endemias, doenças infecto-parasitárias, doenças imunológicas, violência, saúde mental, análises de risco, desiguadades sociais e epidemiologia molecular.

Nesse sentido, há que se divulgar os resultados dos estudos e práticas epidemiológicas nos programas e serviços (incluindo experiências bem sucedidas da vigilância), que possam ser catalogados e disponibilizados por meio impresso, magnético e digital.
Essas experiências compiladas deveriam ter versões completas, organizadas num banco para utilização em treinamentos e capacitações. A Abrasco deve abrir espaços nos congressos para apresentação e debate das experiências que obtiverem resultados mais expressivos, cujos resumos padronizados devem igualmente ser divulgados em sua linha editorial.

Temos de reconhecer que a epidemiologia brasileira é vigorosa e que está em condições de assumir, no âmbito de sua competência, as políticas públicas para o setor nos campos da pesquisa, do ensino e práticas assistenciais.

\section{Anexos}

\section{Anexo 1}

Seminário: III Plano Diretor para o

Desenvolvimento da Epidemiologia no Brasil

02 a 04 de agosto de 2000, Brasília - DF

Expositores

O Ensino da Epidemiologia

Maria Lúcia F. Penna - IMS/Uerj

Marilisa Berti de Azevedo Barros - Unicamp/SP

Moisés Goldbaum - DMP/FM/USP

A Pesquisa em Epidemiologia

César Victora - DMS/FM/UFPel

Maurício Barreto - ISC/UFBA

Reinaldo Guimarães - IMS/UERJ

A Epidemiologia nos Serviços de Saúde

Cláudio Pompeiano de Noronha - SMS/RJ

Jarbas Barbosa da Silva Jr. - Cenepi/FNS/MS

José Cássio de Moraes - Santa Casa/SP 
Anexo 2

Participantes

Alethéa Fagundes Sperb

Amaury Lellis Del Fabbro

Ana Brito

Carlos Henrique Mudado Malleta

Célia Bataglin

César Victora

Chester Luiz Galvão Cesar

Cláudio Pompeiano Noronha

Diana Maul de Carvalho

Eduardo Maia Freese de Carvalho

Expedito Albuquerque Luna

Fabiano Pimenta

Fábio Gomes

Fernando A. Proietti

Guilherme Franco Neto

Gulnar Azevedo Mendonça

Henrique Leonardo Guerra

Jarbas Barbosa

José Cássio de Moraes

José da Rocha Carvalheiro

José Eluf Neto

José Maria Pacheco de Souza

José Wellingtons de Oliveira

Lia Giraldo

Lígia Regina S. Kerr Pontes

Lorene Louise Pinto

Luiz Augusto Facchini

Luiz Roberto Oliveira

Luiza de Marilac Meireles Barbosa

Marcos Drumond

Maria Angélica de Salles Dias

Maria Cristina Boaretto
Divisão de Controle Epidemiológico/GO

DMS/Faculdade de Medicina de Ribeirão Preto/SP

Núcleo de Informação Científica/CpqAM

Superintendência de Vigilância Sanitária/MG

Coordenação de Vigilância Epidemiológica/PR

Depto. de Medicina Preventiva/UFPel

Depto. de Epidemiologia/USP

Depto. de Epidemiologia/SMS/RJ

Depto. de Medicina Preventiva/UFRJ

Depto. de Saúde Coletiva/CpqAM/PE

Coordenação de Vigilância Epidemiológica/Funasa

Cenepi/Funasa

Cenepi/Funasa

Depto. de Medicina Preventiva/UFMG

Coordenação Regional/Funasa

Instituto de Medicina Social/Uerj

Centro de Pesquisas Renée Rachou/CPqRR/MG

Diretor do Cenepi/Funasa

Depto. de Medicina Social/FCMSC/SP

Coordenador dos Institutos de Pesquisa/SP

Depto. de Medicina Preventiva/USP

Faculdade de Saúde Pública/USP

Cenepi/Funasa

Depto. de Saúde Coletiva/CpqAM/PE

Mestrado em Saúde Pública/UFCe

AMP/Famed/UFBA/Sesab

Depto. de Medicina Social/UFPel

Depto. de Saúde Pública/Unesp

Depto. de Epidemiologia/SES/Ce

Proaim/SP

Coordenação de Vigilância Epidemiológica/MG

Secretaria Municipal de Saúde/RJ 


\begin{tabular}{|c|c|}
\hline Maria da Glória Teixeira & Instituto de Saúde Coletiva/BA \\
\hline Maria do Carmo Leal & Depto. de Epidemiologia/Ensp/Fiocruz/RJ \\
\hline Maria Fernanda Lima e Costa & Depto. de Medicina Preventiva/CPqRR/MG \\
\hline Maria Lúcia Carneloso & Depto. de Epidemiologia/SES/GO \\
\hline Maria Lúcia Penna & Instituto de Medicina Social/Uerj \\
\hline Maria Regina Fernandes & Coade/Cenepi/Funasa \\
\hline Marlene Tavares B. de Carvalho & Depto. de Epidemiologia/SES/BA \\
\hline Marilisa Berti de Azevedo Barros & $\begin{array}{l}\text { Depto. de Medicina Preventiva e Social/FCM/ } \\
\text { Unicamp }\end{array}$ \\
\hline Maurício Gomes Pereira & Depto. de Saúde Coletiva/UNB \\
\hline Maurício Lima Barreto & Instituto de Saúde Coletiva/UFBA \\
\hline Michael Reicheinhein & Instituto de Medicina Social/UERJ \\
\hline Moisés Goldbaum & Depto. de Medicina Preventiva/USP \\
\hline Myriam Debert Ribeiro & Depto. de Medicina Preventiva/Unifesp \\
\hline Naomar de Almeida Filho & Instituto de Saúde Coletiva/UFBA \\
\hline Otaliba Libâno de Morais Neto & Depto. de Medicina Tropical/UFGO/DTPS \\
\hline Paulo Roberto de Almeida Barbosa & Depto. de Doenças Imunopreviníveis/SES/RJ \\
\hline Pedro Miguel dos Santos Neto & RH/Vigisus/Cenepi/Funasa/MS \\
\hline Pedro Tauil & Depto. de Saúde Coletiva/UNB \\
\hline Reinaldo Guimarães & Instituto de Medicina Social/Uerj \\
\hline Ricardo Ximenes & UFPE \\
\hline Rita Barradas Barata & Depto. de Medicina Social/FCMSC/SP \\
\hline Roseli Cerqueira & Cenepi/Funasa \\
\hline Sérgio Koifman & Depto. de Epidemiologia/Ensp/Fiocruz \\
\hline Therezinha de Almeida Aquino & Depto. de Epid. e Vigilância da Saúde/SMS/RE \\
\hline Vera Gattás & Cenepi/Funasa \\
\hline Waltair Maria Martins Pereira & Depto. de Epidemiologia/SES/PA \\
\hline Zulmira Maria de Araújo Hartz & Fiocruz \\
\hline Bárbara Marques & Secretária - Abrasco \\
\hline
\end{tabular}

